\title{
A robust monitoring technique for fault detection in grid-connected PV plants
}

\author{
${ }^{1}$ Fouzi Harrou, Member, IEEE, ${ }^{2,3}$ Bilal Taghezouit \\ ${ }^{1}$ Ying Sun \\ ${ }^{1}$ King Abdullah University of Science and Technology (KAUST) ${ }^{2}$ Centre de Développement des Energies Renouvelables \\ CEMSE Division \\ Thuwal, 23955-6900, Saudi Arabia \\ fouzi.harrou@kaust.edu.sa \\ CDER, B.P. 62, Route de l'Observatoire, Bouzaréah, \\ and ${ }^{3}$ Ecole Nationale Polytechnique Alger, \\ Laboratoire de Dispositif de Communication \\ et de Conversion Photovoltaique, Algiers, Algeria
}

\begin{abstract}
Monitoring the operation condition of photovoltaic (PV) systems is crucial to improving their efficiency. In this paper, an effective method to supervise the DC part of PV plants under noisy environment is provided. In fact, noisy measurements make the supervision more challenging as the feature extraction of the fault is more difficult. The designed approach merges the desirable proprieties of the discrete wavelet transform and the exponentially weighted moving average scheme to appropriately detect faults in PV system. Specifically, this approach is employed to check the residuals generated by a simulation model based on a single-diode modeling for fault detection. We evaluated the efficiency of the proposed approach on a real PV system in Algeria. Results indicated that the proposed approach has good capacity in supervising the DC part of PV plants.
\end{abstract}

\section{INTRODUCTION}

Efficiency and reliability of PV systems are achieved by optimizing the PV systems performance. However, PV plants are exposed to numerous circumstances such as shading and failure that affect their performance. In particular, anomalies and failures affecting the DC part of PV plants are usually challenging to avoid and can generate energy loss and even severe safety concerns [1], [2]. Accordingly, fault detection in PV plants is an important component to keeping high efficiency, safety and reducing maintenance costs [3]-[5].

Automatic detection of potential anomalies becomes vital for supervising the operation of PV systems [6], [7]. The aim of fault detection techniques is identifying the dissimilarity between the designed and actual performances of the inspected PV plant. Fault detection mechanisms provide relevant information by detecting degradation and providing protection against equipment failure and thus reduce the maintenance cost. For instance, faults in DC side of PV array if not detected in time, they can cause to serious problems such as fire hazards [1], [2]. To bypass the drop of the PV system yield, numerous detection techniques were developed in the literature including model-based procedures [4], [8], Kalman filter [9], and spectral methods [10]. Other methodologies use machine learning mechanisms [11]-[13], like support vector machine [14], k-nearest neighbors [15], decision tree procedure [16], Bayesian neural network [11] and fuzzy logic [13]. Many other interesting monitoring mechanisms were discussed in the literature can be found in [17],
[18]. These approaches necessitate the presence of relevant measurements describing the system performances under normal and faulty situations [4].

In other studies, statistical monitoring techniques are used to supervise the operation of PV systems. In [19], a method to detect faults in a PV plant without a need for meteorological data is proposed. The detection is conducted by analyzing the output energy of the PV plant via the analysis of variance test. In [5], a model-based method to detect faults in PV system is introduced. This method uses the Multivariate CUmulative SUM procedure to evaluate the residuals from the PV simulation model for monitoring the operation of a PV plant. Braun et al. [20] aimed to monitor PV plants using multivariate outlier rules. This approach uses current and voltage measurements of each separate PV panel for fault detection. Platon et al. [21] presented three-sigma-based approach for online fault detection in PV plants. It is known that the three-sigma-based schemes are effective in detecting large faults, but ineffective in detecting incipient faults. In [4], [22], a monitoring approach has been presented to detect temporary shading and faults occurred in the DC side of PV systems. This technique aims to improve detection of incipient faults in PV systems based on exponentially weighted moving average (EWMA) approach. However, EWMA-based approaches don't consider the multiresolution nature of measurements. Furthermore, measurements noise can hide important features in data or lead to misleading indications by increasing false alarms and missed detections. Multiresolution representation of data based on wavelets is an efficient feature extraction tool that has the ability to feature/noise separation and decorrelating time series data [23]-[26]. Theretofore, the aim in this work is to merge the benefits of multiscale representation based on wavelet and of an EWMA scheme to deal with the problem of measurement noise in the data and to improve fault detection in PV plants. The proposed mechanism will be termed WM-EWMA hereafter. Specifically, the WM-EWMA approach is employed to check the residuals generated by the simulated model to detect faults. Performance of this monitoring mechanism has been evaluated via real data from a grid-connected plant in Algeria. Results suggest that the 
detection performance can be effectively improved by using the proposed approach compared to the conventional EWMA chart.

\section{PV MODULE MODELING}

Solar cell/behavior is commonly modeled by a single diode model (SDM) as depicted in Figure 1.

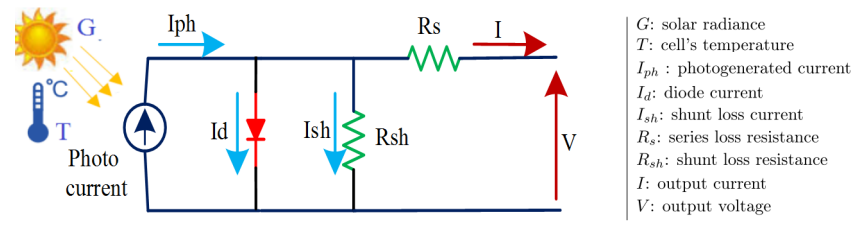

Fig. 1. SDM of solar cells and PV modules.

The analytic expression that describes the I-V characteristics of a PV cell/module based on the model shown in Figure 1 is:

$$
I=I_{p h}-\underbrace{I_{0}\left[\exp \left(\frac{q\left(V+R_{s} I\right)}{n k_{B} T}\right)-1\right]}_{I_{d}}-\underbrace{\frac{V+R_{s} I}{R_{s h}}}_{I_{s h}},
$$

where $I_{0}$ is the dark saturation current, $n$ is the diode's ideality factor, $k_{B}$ is Boltzmann's constant, and $q$ represents the electronic charge. The precision of SDM in Equation (1) is related to the selected values of the unknown parameters $I_{p h}, I_{0}, n, R_{s}$ and $R_{s h}$.

Generally speaking, the construction of a simulation model describing the nominal operating conditions of the inspected PV system conducted in two steps: (a) identify the unknown parameters of SDM, and (b) construct a model describing the performances of the real PV plant (see Figure 2).

Firstly, the optimal values of the unknown parameters of the SDM are determined using a heuristic algorithm and (I-V) curve measurements. Specifically, the Artificial Bee Colony (ABC) algorithm selects the optimal values of the SDM parameters that minimize the difference between the measured and the estimated I-V curves [4], [27].

$$
R M S E=\sqrt{\frac{I}{m} \sum_{i=1}^{m}\left[f_{i}\left(I_{\text {meas }}, V_{\text {meas }}, \theta\right)\right]^{2}},
$$

where

$$
\begin{array}{r}
f\left(I_{\text {meas }}, V_{\text {meas }}, \varphi\right)=I_{\text {meas }}-\left\{I_{\text {ph }}-\right. \\
\left.I_{0}\left[\exp \left(\frac{q\left(V_{\text {meas }}+R_{s} I_{\text {meas }}\right)}{n k_{B} T}\right)-1\right]-\frac{V_{\text {meas }}+R_{s} I_{\text {meas }}}{R_{\text {sh }}}\right\}
\end{array}
$$

$\varphi$ represent the vector of unknown parameters $\left[I_{p h}, I_{0}\right.$, $n, R_{s}$ and $\left.R_{s h}\right]$. Finally, to simulate the entire PV array, a co-simulation between Matlab/Simulink and PSIM is implemented for simulating the inspected PV system by using the computed SDM parameters.

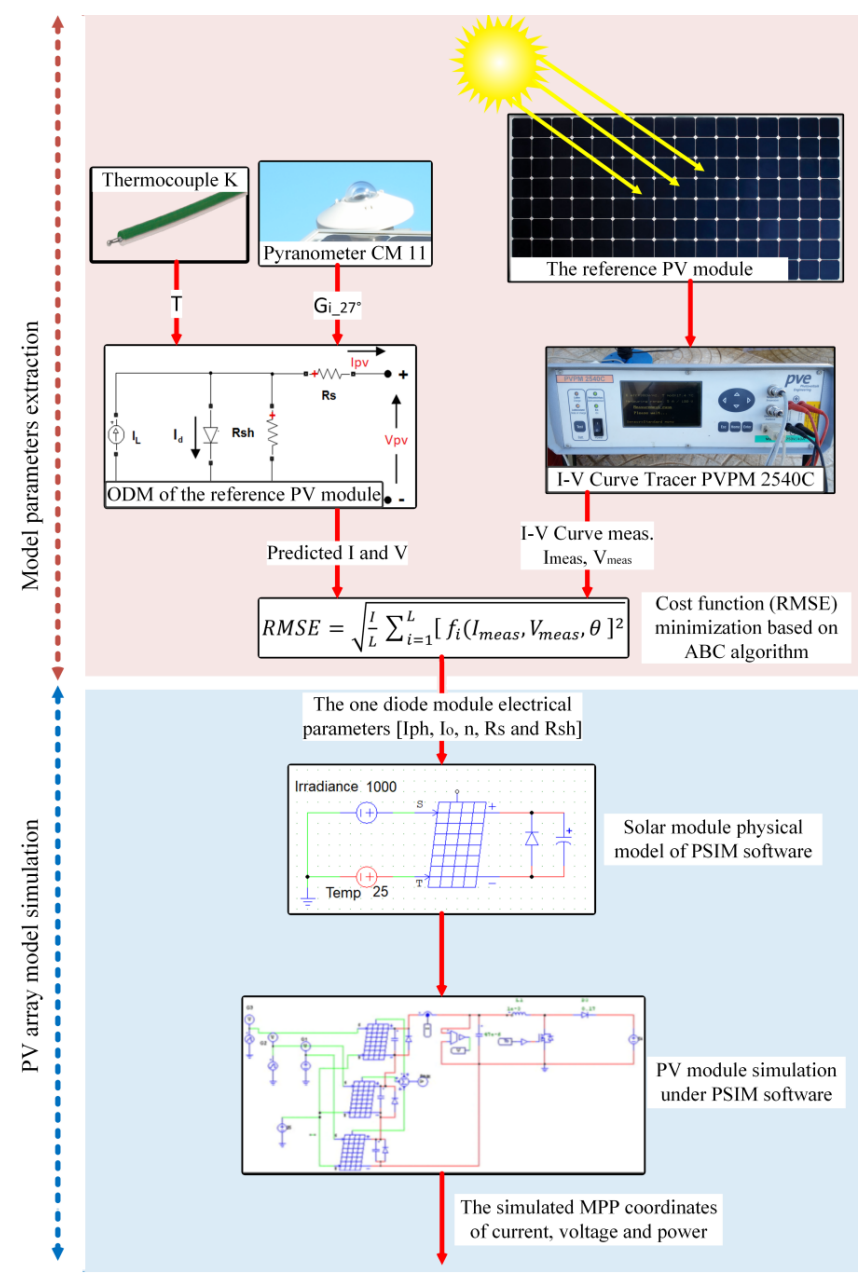

Fig. 2. Illustration of the PV modeling framework.

\section{PRoposed MW-EWMA MONITORING MECHANISM}

For monitoring the DC part of PV plants, residuals representing the mismatch between the real measurements $\left(I_{t}\right.$, $V_{t}$ and $\left.P_{t}\right)$ and estimation of MPP current $\left(\hat{I}_{t}\right)$, MPP voltage $\left(\hat{V}_{t}\right)$ and MPP power $\left(\hat{P}_{t}\right)$ using the simulation model are fed to the developed MW-EWMA monitoring scheme for fault detection.

$$
\tilde{I}_{t}=I_{t}-\hat{I}_{t}, \quad \tilde{V}_{t}=V_{t}-\hat{V}_{t}, \quad \tilde{P}_{t}=P_{t}-\hat{P}_{t}, \quad t \in[1, n],
$$

The MW-EWMA mechanism is used to assess the residuals to uncover faults.

\section{A. EWMA scheme}

EWMA scheme is an important univariate quality control procedure, which has been used in several disciplines to online monitor the mean of production processes [28], [29], [29], [30]. Here, some background information on EWMA is presented. The EWMA fault decision statistic is computed as follows [28]:

$$
z_{t}=\nu x_{t}+(1-\nu) z_{t-1}, \quad z_{0}=\mu_{0},
$$


where $x_{t}$ is the measurement at time instant $t$, the initial value, $z_{0}$ is the fault-free mean of data, $\mu_{0}$, and $\nu(0<\nu \leq 1)$ is a smoothing parameter. The thresholds of the EWMA are computed as,

$$
U, L=\mu_{0} \pm 3 \sigma_{0} \sqrt{\frac{\nu}{(2-\nu)}\left[1-(1-\nu)^{2 t}\right]},
$$

where $\sigma_{0}$ represent the standard deviation of the collected data under nominal conditions. The EWMA mechanism delivers a signal of a fault if $z_{t}$ overpass the decision thresholds.

\section{B. WM-EWMA approach for PV system monitoring}

Multiscale representation of data using wavelet allows simultaneous time and frequency analysis of data. It has been used in broad applications due to its decorrelation property and its capability in separating feature/noise. By applying the Discrete Wavelet Transform (DWT), a signal, $x(t)$, can be represented as a combination of approximation, $A_{J}(t)$ and detail coefficients, $D_{j}(t)[31]$.

$$
x(t)=A_{J}(t)+\sum_{j=1}^{J} D_{j}(t)
$$

where the coarsest scale $J$ usually called the decomposition level.

The proposed WM-EWMA fault detection mechanism integarates the benefits of multiscale representation with those of the EWMA approach. Specifically, in WM-EWMA mechanism, the residuals from the PV simulation model are decomposed using DWT. In training phase, a fault-free training data set was utilized to compute the EWMA thresholds at multiple scales. In the training phase, the EWMA thresholds computed previously are applie to the detailed signals of the testing data on multiple scales for fault detection. This approach is outlined in Algorithm 1.

\section{RESULTS AND DISCUSSION}

We evaluate the developed fault detection mechanism usng real data from a PV system located in Algeria. This sysetm consists of a PV array of 90 modules type Isofoton I-106/12 providing a total peak power of $9.6 \mathrm{kWp}$. The PV array is made up of three identical PV sub-array of $3.2 \mathrm{kWp}$. Each sub-array is comprised of 2 branches in parallel of $15 \mathrm{PV}$ modules in series. The main components of the supervised PV sub-system are illustrated in Figure 4. $\overline{\text { Algorithm } 1 \text { PV system monitoring via WM-EWMA scheme }}$

\section{1) Training stage:}

Step 1 Collect the training data set (fault-free data), representative of a nominal situation. This training data is necessary to set the control limits.

Step 2 Build a simulation model for the inspected PV array.

Step 3 Compute the residuals using the simulation model.

Step 4 Decompose the residuals into wavelet coefficients using DWT. This changes the data set from a single scale set into a multiple scales set, which can allow for multiscale monitoring.

Step 5 Compute the EWMA statistic and the control limits.

Step 6 Keep only scales that have threshold violations.

Step 7 Reconstruct data using only retained scales.

Step 8 Compute EWMA control limits for reconstructed data.

\section{2) Testing stage (test the new data):}

Step 1 Generate residuals using the constructed simulation model based on the testing data.

Step 2 Decompose the residuals into multiple scales.

Step 3 Compute the EWMA statistic and utilize the control limits for each scale obtained in the training stage.

Step 4 Retain only coefficients that violate the threshold; neglect other coefficients.

Step 5 Reconstruct the signal using only retained coefficients.

Step 6 Report a fault when the WM-EWMA statistic exceeds the control limits obtained from training stage.

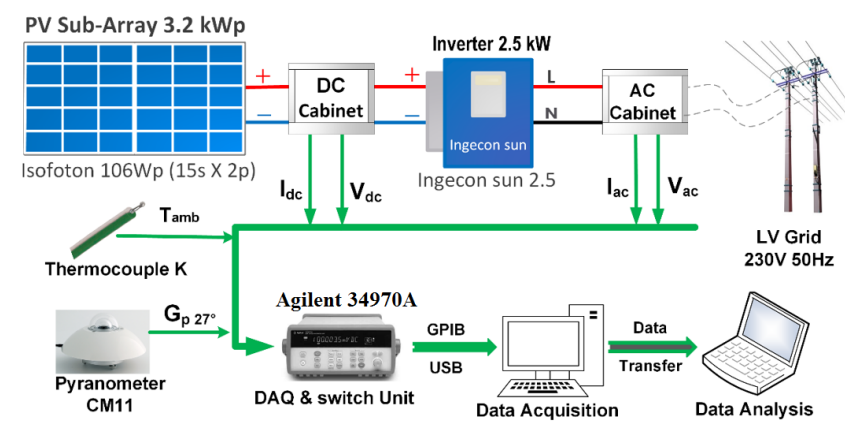

Fig. 4. Schematic representation of the inspected PV subsystem with data acquisition.

By applying the ABC optimization procedure, we determined the optimal values of the parameters $\left[I_{p h}^{*}, I_{0}^{*}, n^{*}, R_{s}^{*}, R_{s h}^{*}\right]$, which provide the suitable fitting measured (I-V) characteristics (Table I). As described above,

TABLE I

COMPUTED MODULE PARAMETERS OF THE SDM VIA THE BEST-SO-FAR ABC PROCEDURE.

\begin{tabular}{l|l|l|l|l|l|l}
\hline & $I_{p h}[\mathrm{~A}]$ & $I_{0}[\mathrm{~A}]$ & $n$ & $R_{s}[\Omega]$ & $R_{s h}[\Omega]$ & $\mathrm{RMSE}$ \\
\hline ISOFOTON 106-12 & 6.54 & $1.11 \mathrm{e}-0.5$ & 1.66 & 0.1474 & 202.6 & 0.014 \\
\hline
\end{tabular}

a co-simulation of Matlab/Simulink with PSIM is used to 


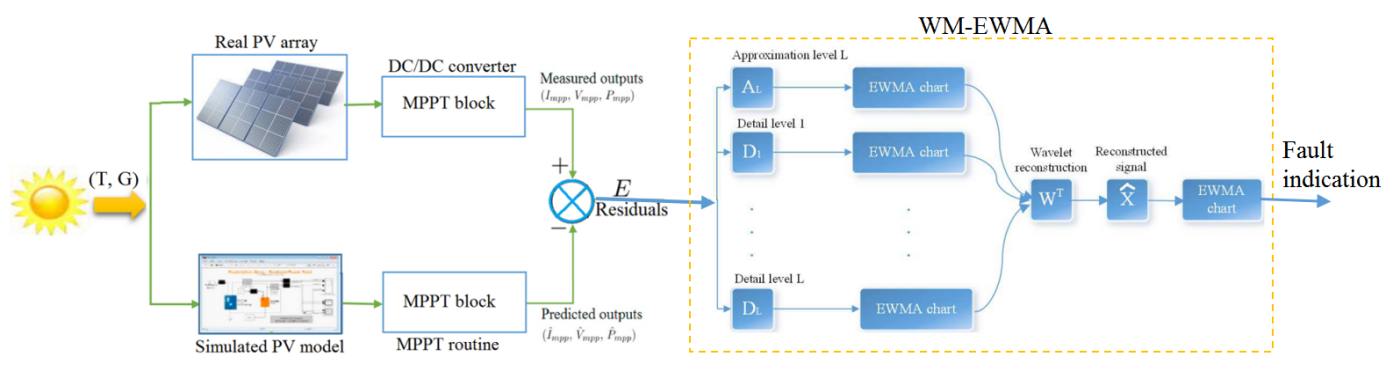

Fig. 3. Multiscale EWMA monitoring framework.

simulate the inspected PV array based on the determined SDM parameters. Figure 5) displays the measured and estimated MPP current measurement using the simulation model when introducing three real days of temperature and irradiances into the model. Figure 5 indicates the good agreement between the actual measurements and the model output.

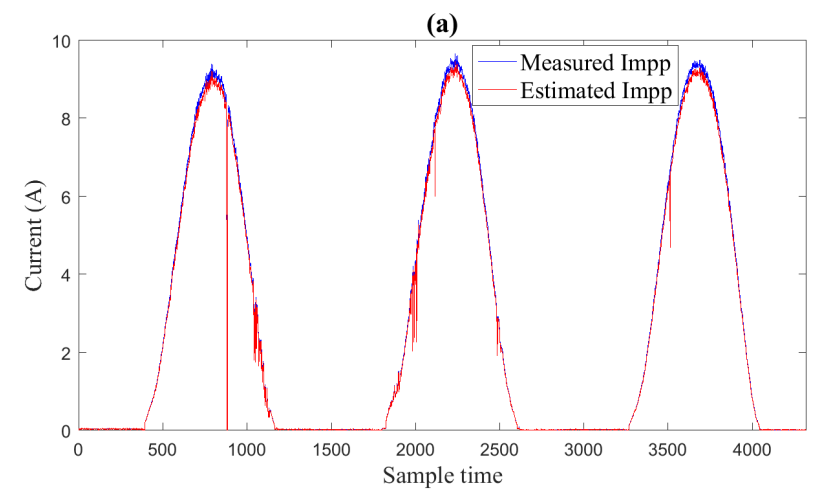

Fig. 5. Measurement and MPP current prediction form the simulation model

Now, we evaluate the the WM-EWMA mechanism in detecting open-circuit faults in a PV array under noisy conditions. In this experiment, the first string is disconnected from the inspected PV array for time instant 300 to 500 . To demonstrate the efficiency of the proposed mechanism, in this case study, we add random noises with different levels following the normal distributions to measured data.

Results of EWMA and WM-EWMA procedures are displayed in Figures 6 and Figures 7 for the cases of moderate $(\sigma=0.8)$ and highly noisy measurements $(\sigma=$ $1.6)$, respectively.From this scenario, it can be seen that the WM-EWMA is more robust to noise measurements than the EWMA scheme (see Figures 6 and Figures 7).

The performance of both techniques in term of false alarm rate (FAR) and missed detection rate (MDR) are given in Table II. Results in Table II confirms the superiority and robustness to noise measurement of the WM-EWMA approach compared to conventional EWMA approach. it is worthnoting that highly noisy measurements have an impact on the performance of the EWMA chart, and the impact seems less serious for the proposed MW-EWMA chart.

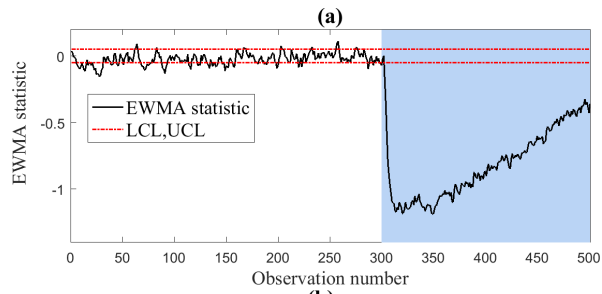

(b)

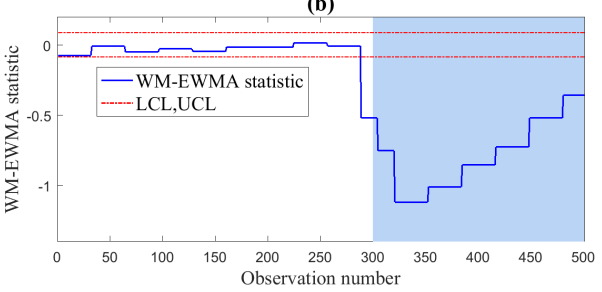

Fig. 6. (a) EWMA and (b) WM-EWMA mechanisms in the case of an open-circuit fault, moderate noisy data scenario.

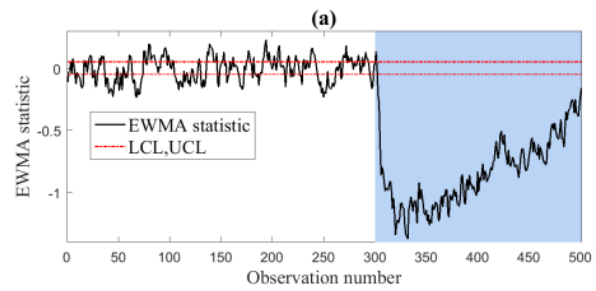

(b)

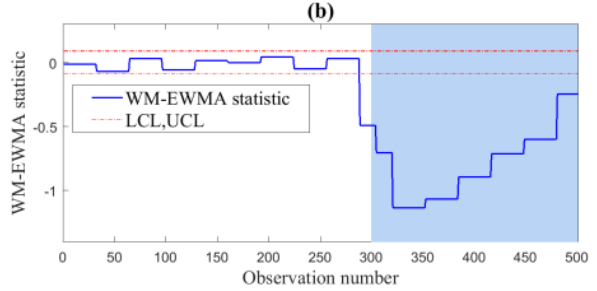

Fig. 7. (a) EWMA and (b) WM-EWMA mechanisms in the case of an open-circuit fault, highly noisy data scenario.

TABLE II

PERFORMANCES OF THE EWMA AND MW-EWMA MECHANISMS.

\begin{tabular}{|c|c|c||c|c|}
\hline \multicolumn{3}{|c|}{ EWMA chart } & \multicolumn{2}{c|}{ WM-EWMA chart } \\
\hline$\sigma$ & FAR & MDR & FAR & MDR \\
\hline 0.2 & 11.34 & 0 & 4.10 & 0 \\
\hline 0.8 & 34.33 & 0.98 & 4.70 & 0 \\
\hline 1.6 & 66 & 0.5 & 5 & 0 \\
\hline
\end{tabular}

\section{CONCLUSION}

This article has focused on monitoring the DC side of PV systems under noisy environment. An efficient fault detection 
strategy merging the multiresolution representation of data using wavelets and EWMA scheme is proposed in this study for monitoring the operating conditions of PV systems. In particular, the developed approach is conducted in two steps: (a) construct a simulation model for the inspected PV system based on single diode PV model, (b) generate residuals from the simulation model using new test data and (c) apply the fault detection approach to the uncorrelated residuals to uncover possible faults. Real data from a $9.54 \mathrm{kWp}$ PV plant in Algeria has been used for evaluating the performance of the proposed approach. Results show the efficiency of the proposed scheme in reliably detecting faults in DC side of PV systems even under noisy conditions.

\section{ACKNOWLEDGMENT}

This publication is based upon work supported by the King Abdullah University of Science and Technology (KAUST) Office of Sponsored Research (OSR) under Award No: OSR-2015-CRG4-2582.

\section{REFERENCES}

[1] B. Brooks, "The bakersfield fire-A lesson in ground-fault protection," SolarPro Mag, pp. 62-70, 2011.

[2] M. K. Alam, F. Khan, J. Johnson, and J. Flicker, "A comprehensive review of catastrophic faults in PV arrays: types, detection, and mitigation techniques," IEEE Journal of Photovoltaics, vol. 5, no. 3, pp. 982-997, 2015.

[3] F. Harrou, Y. Sun, B. Taghezouit, A. Saidi, and M.-E. Hamlati, "Reliable fault detection and diagnosis of photovoltaic systems based on statistical monitoring approaches," Renewable Energy, vol. 116, pp. 22-37, 2018

[4] E. Garoudja, F. Harrou, Y. Sun, K. Kara, A. Chouder, and S. Silvestre, "Statistical fault detection in photovoltaic systems," Solar Energy, vol. 150, pp. 485-499, 2017.

[5] F. Harrou, Y. Sun, and A. Saidi, "Model-based fault detection algorithm for photovoltaic system monitoring," in Computational Intelligence (SSCI), 2017 IEEE Symposium Series on. IEEE, 2017, pp. 1-5.

[6] X. Li, H. Wen, G. Chu, and Z. Ye, "A novel PV faults diagnosis method based on the structure of differential power processing," in Renewable Energy Research and Applications (ICRERA), 2017 IEEE 6th International Conference on. IEEE, 2017, pp. 685-689.

[7] T. de Carvalho Rêgo, V. L. D. Ferreira, J. B. Parizzi, and F. L. Dias, "Detection of transients in photovoltaic signals via high order statistics," in Renewable Energy Research and Applications (ICRERA), 2017 IEEE 6th International Conference on. IEEE, 2017, pp. 931-936.

[8] K.-H. Chao, S.-H. Ho, and M.-H. Wang, "Modeling and fault diagnosis of a photovoltaic system," Electric Power Systems Research, vol. 78, no. 1, pp. 97-105, 2008.

[9] B.-K. Kang, S.-T. Kim, S.-H. Bae, and J.-W. Park, "Diagnosis of output power lowering in a PV array by using the kalman-filter algorithm," IEEE Transactions on Energy Conversion, vol. 27, no. 4, pp. 885-894, 2012.

[10] J. Johnson, S. Kuszmaul, W. Bower, and D. Schoenwald, "Using PV module and line frequency response data to create robust arc fault detectors," in Proceedings of the 26th European Photovoltaic Solar Energy Conference and Exhibition, 2011, pp. 05-09.

[11] A. M. Pavan, A. Mellit, D. De Pieri, and S. Kalogirou, "A comparison between BNN and regression polynomial methods for the evaluation of the effect of soiling in large scale photovoltaic plants," Applied energy, vol. 108, pp. 392-401, 2013.

[12] H. Mekki, A. Mellit, and H. Salhi, "Artificial neural network-based modelling and fault detection of partial shaded photovoltaic modules," Simulation Modelling Practice and Theory, vol. 67, pp. 1-13, 2016.

[13] M. Tadj, K. Benmouiza, A. Cheknane, and S. Silvestre, "Improving the performance of PV systems by faults detection using GISTEL approach,' Energy conversion and management, vol. 80, pp. 298-304, 2014.
[14] Z. Yi and A. H. Etemadi, "Line-to-line fault detection for photovoltaic arrays based on multiresolution signal decomposition and two-stage support vector machine," IEEE Transactions on Industrial Electronics, vol. 64, no. 11, pp. 8546-8556, 2017.

[15] S. R. Madeti and S. Singh, "Modeling of pv system based on experimental data for fault detection using knn method," Solar Energy, vol. 173, pp. 139-151, 2018.

[16] R. Benkercha and S. Moulahoum, "Fault detection and diagnosis based on c4. 5 decision tree algorithm for grid connected pv system," Solar Energy, vol. 173, pp. 610-634, 2018.

[17] A. Triki-Lahiani, A. B.-B. Abdelghani, and I. Slama-Belkhodja, "Fault detection and monitoring systems for photovoltaic installations: A review," Renewable and Sustainable Energy Reviews, vol. 82, pp. 2680-2692, 2017.

[18] A. Mellit, G. M. Tina, and S. A. Kalogirou, "Fault detection and diagnosis methods for photovoltaic systems: A review," Renewable and Sustainable Energy Reviews, vol. 91, pp. 1-17, 2018.

[19] S. Vergura, "Hypothesis tests-based analysis for anomaly detection in photovoltaic systems in the absence of environmental parameters," Energies, vol. 11 , no. 3, p. 485, 2018.

[20] H. Braun, S. Buddha, V. Krishnan, A. Spanias, C. Tepedelenlioglu, T. Yeider, and T. Takehara, "Signal processing for fault detection in photovoltaic arrays," in IEEE International Conference on Acoustics, Speech and Signal Processing (ICASSP). IEEE, 2012, pp. 1681-1684.

[21] R. Platon, J. Martel, N. Woodruff, and T. Y. Chau, "Online fault detection in PV systems," IEEE Transactions on Sustainable Energy, vol. 6, no. 4, pp. 1200-1207, 2015.

[22] F. Harrou, Y. Sun, B. Taghezouit, A. Saidi, and M.-E. Hamlati, "Reliable fault detection and diagnosis of photovoltaic systems based on statistical monitoring approaches," Renewable Energy, vol. 116, pp. 22-37, 2018

[23] F. Harrou, M. Madakyaru, and Y. Sun, "Improved nonlinear fault detection strategy based on the hellinger distance metric: Plug flow reactor monitoring," Energy and Buildings, vol. 143, pp. 149-161, 2017.

[24] M. Madakyaru, F. Harrou, and Y. Sun, "Improved anomaly detection using multi-scale PLS and generalized likelihood ratio test," in Computational Intelligence (SSCI), 2016 IEEE Symposium Series on. IEEE, 2016, pp. 1-6.

[25] F. Harrou, M. Nounou, and H. Nounou, "Enhanced monitoring using pca-based glr fault detection and multiscale filtering," in Computational Intelligence in Control and Automation (CICA), 2013 IEEE Symposium on. IEEE, 2013, pp. 1-8.

[26] M. Sheriff, F. Harrou, and M. Nounou, "Univariate process monitoring using multiscale Shewhart charts," in International Conference on Control, Decision and Information Technologies (CoDIT). IEEE, 2014, pp. 435-440.

[27] D. Karaboga and B. Basturk, "On the performance of artificial bee colony (ABC) algorithm," Applied soft computing, vol. 8, no. 1, pp. 687-697, 2008.

[28] D. C. Montgomery, "Introduction to statistical quality control," John Wiley\& Sons, New York, 2005.

[29] F. Harrou, Y. Sun, M. Madakyaru, and B. Bouyedou, "An improved multivariate chart using partial least squares with continuous ranked probability score," IEEE Sensors Journal, vol. 18, no. 16, pp. 6715-6726, 2018.

[30] F. Harrou and M. Nounou, "Monitoring linear antenna arrays using an exponentially weighted moving average-based fault detection scheme," Systems Science \& Control Engineering: An Open Access Journal, vol. 2, no. 1, pp. 433-443, 2014

[31] R. Gao and R. Yan, Wavelets: Theory and applications for manufacturing. Springer Science \& Business Media, 2010. 